\title{
Conductance oscillation of a mesoscopic normal metal spanning unconventional and conventional superconductors
}

\author{
Junren Shi, Jinming Dong, and D. Y. Xing \\ Department of Physics and Laboratory of Solid State Microstructure, Nanjing University, Nanjing 210008, \\ People's Republic of China \\ Z. D. Wang \\ Department of Physics, University of Hong Kong, Pokfulam Road, Hong Kong, People's Republic of China
}

(Received 2 July 1997)

\begin{abstract}
We present a theory for the conductance of a mesoscopic normal metal spanning two superconductors, in which an analytical expression of the conductance is formulated. It is found that the conductance oscillates with the phase difference of two superconductors periodically. When one of the superconductors has a $d$-wave symmetry, the $2 \pi$-period component of the conductance oscillation decays with the misorientation angle $\alpha$ and vanishes at $\alpha=\pi / 4$ in contradiction to the $s$-wave case, from which a method is proposed to identify unambiguously the pairing symmetry of the high- $T_{c}$ superconductors. [S0163-1829(97)04745-0]
\end{abstract}

Since the discovery of high- $T_{c}$ superconductors, much effort has been devoted to make clear the pairing mechanism and pairing symmetry of these superconductors. Although the experiment of NMR relaxation rate restricts the pairing states to be of $s$-wave or $d$-wave symmetry, ${ }^{1}$ the issue remains controversial. Based on various pairing models, the $d_{x^{2}-y^{2}}$ symmetry, ${ }^{2,3}$ the anisotropic $s$-wave state, ${ }^{4}$ and other pairing states mixing $d$-wave and $s$-wave symmetry ${ }^{5-7}$ have been suggested. So it is crucial now to determine the pairing symmetry of the high- $T_{c}$ superconductors unambiguously for understanding their underlying microscopic mechanism.

The common feature of the unconventional pairing states is their anisotropic order parameters. Generally, there are two kinds of anisotropies: magnitude and phase. The magnitude anisotropy exists in all proposed unconventional pairing states, and is shown in the excess quasiparticle excitations caused by reduced gap in some directions, which strongly affects the low-temperature transport and tunnelling spectra. Nevertheless, most experiments of the magnitude anisotropy ${ }^{8-10}$ could not be used to distinguish, e.g., the anisotropic $s$-wave state from the $d$-wave one, and sometimes even reach contradictory conclusions. On the other hand, the phase anisotropy exists in the extended $s$-wave state, the $d_{x^{2}-y^{2}}$ state, as well as some mixture states, but is absent in both isotropic and anisotropic $s$-wave states. In the $d_{x^{2}-y^{2}}$ state with order parameter $\Delta(\mathbf{k})=\Delta_{d}\left(k_{a}^{2}-k_{b}^{2}\right)$, where $k_{a}$ and $k_{b}$ are the unit wave vectors along the $a$ axis and $b$ axis in the $\mathrm{CuO}_{2}$ plane, respectively, those quasiparticles with $\left|k_{a}\right|<\left|k_{b}\right|$ will experience a negative order parameter and so obtain an additional $\pi$ phase shift relative to those with $\left|k_{a}\right|>\left|k_{b}\right|$. However, for the pairing states with $s$-wave symmetry, including the anisotropic $s$-wave state, the order parameters are always positive, and there is no any additional phase shift. A $\pi$ phase shift observed in a corner superconducting quantum interference device (SQUID) is a clear indication of the phase anisotropy favoring the $d$-wave symmetry, ${ }^{11,12}$ which is supported by some experiments, ${ }^{13}$ but not by others. ${ }^{8,9}$ In order to find a way to identify the pairing symmetry of the high- $T_{c}$ superconductors, current theoretical works ${ }^{14-16}$ pay much attention to determining the phase anisotropy. Notice that mixing effects of both magnitude and phase anisotropies existed in these theoretical works and most experiments make the determination of the phase symmetry and phase distribution much more complicated.

In this paper, we propose a method to identify the pairing symmetry of the high- $T_{c}$ superconductors, which is sensitive to the different kinds of pairing states. More importantly, it is independent of the magnitude anisotropy and relates only to the phase anisotropy. Using this method, we can in principle determine the phase distribution of the order parameter. The idea originates from a recent experiment made by de Vegvar et al. ${ }^{17}$ in a superconductor-normal-metal-superconductor (SNS) structure composed of one normal metal (an Au metal wire) spanning two conventional superconductors $(\mathrm{Nb})$ shown in Fig. 1. They studied the conductance oscillation of the normal metal by adjusting the phase difference $\varphi$ between two superconductors, and found that the conductance oscillates periodically with $\varphi$ by a period $2 \pi$. The conductance oscillation of a cross structure in contact with two conventional superconductors was also reported. ${ }^{18}$ The phenom-

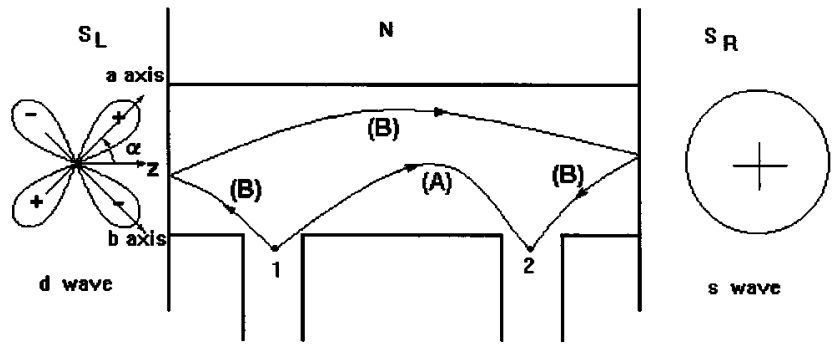

FIG. 1. The structure of the system. A normal mesoscopic metal spanning between two superconductors in the regime $\xi_{c}$, $\xi_{N} \ll d_{N} \sim L_{\varphi}$. The conductance is measured between lead 1 and 2 . (A) and (B) represent two kinds of paths, both contributing to the transmission coefficient between the lead 1 and 2 . 
enon can be understood by the Andreev reflection. ${ }^{19,20}$ According to the Landauer-Büttiker's formula, ${ }^{21,22}$ the normal metal conductance is given by $\left(2 e^{2} / h\right) T_{t}$, where $T_{t}$ is the transmission coefficient from lead 1 to lead 2. Two different Feynman paths shown in Fig. 1 interfere with each other, both making contributions to $T_{t}$. The path (A) indicates a direct propagation of electron from lead 1 to lead 2. For path (B), an electron propagates to the left SN interface first, undergoing an Andreev reflection into a hole and acquiring a phase shift $\varphi_{L}$. Then the hole diffuses to the right $\mathrm{SN}$ interface, undergoing again an Andreev reflection into an electron and acquiring another phase shift $-\varphi_{R}$. After the electron arrives at lead 2 finally, its additional phase shift should be $\varphi=\varphi_{L}-\varphi_{R}$. Owing to the interference effects of two paths, the conductance will oscillate with $\varphi$, which can be controlled externally.

Now, if one of the conventional superconductor in the SNS structure is replaced by a phase-anisotropic one, the relative phase shift of the unconventional superconductor makes a contribution to the total phase shift of the path (B), which will lead to an observable difference in the phase dependence of the conductance oscillation for different pairing symmetries.

In the following, we will first derive the conductance formula of a mesoscopic normal metal in a SNS structure. As a test for the formula, we will compare our result with the experiment done on the conventional superconductors. Then the effects of the unconventional pairing states will be treated. Finally the nonideal effects will be considered.

In the structure, the length $d_{N}$ of the normal wire should satisfy the condition $\xi_{c}, \xi_{N} \ll d_{N}<L_{\varphi}$, where $\xi_{c}$ is the Cooper-pair coherence length, $\xi_{N}$ is the characteristic distance of the proximity effect, and $L_{\varphi}$ is the phase-relaxation length of the normal metal. In this case, Josephson current could be neglected.

In our system, the electrons are confined between two SN interfaces. At very low temperatures, only the electrons near the Fermi surface have contributions to the conductivity of the normal metal, which is mainly determined by the property of the bound states near the Fermi surface, and the effect due to the scattering states is negligible. As usual, if we consider the SN interfaces to be ideal, the pair potential $\Delta_{N}$ in the normal metal region should be zero. The pair potentials of the left and right superconductors are expressed as $\left|\Delta_{L}(\theta, \phi)\right| \exp \left[i \psi_{L}(\theta, \phi)+i \varphi_{L}\right]$ and $\left|\Delta_{R}(\theta, \phi)\right| \exp \left[i \psi_{R}(\theta, \phi)\right.$ $\left.+i \varphi_{R}\right]$, respectively. Here, the $z$ axis is taken along the normal of the interface, $\theta$ and $\phi$ are the polar angle and azimuthal angle, respectively, $\varphi_{L}$ and $\varphi_{R}$ are the external phases of the left and right superconductors, respectively, $\psi_{L}(\theta, \phi), \psi_{R}(\theta, \phi)$ are the relative phase shifts in the unconventional pairing states, which satisfy $\exp \left[i \psi_{L(R)}(\theta, \phi)\right]=\Delta_{L(R)}(\theta, \phi) /\left|\Delta_{L(R)}(\theta, \phi)\right|$, and are zero in the isotropic and anisotropic $s$-wave cases.

The conductance is calculated using a Landauer-type conductance formula ${ }^{23}$

$$
g=\frac{e^{2}}{h} \sum_{n} \int_{-\infty}^{\infty} d \epsilon \frac{\partial f(\epsilon)}{\partial \epsilon} \frac{\Gamma_{L n}(\epsilon) \Gamma_{R n}(\epsilon)}{\Gamma_{L n}(\epsilon)+\Gamma_{R n}(\epsilon)} \operatorname{Im} G_{n}^{r}(\epsilon),
$$

and the sum is taken over all states in the normal metal. Here, $\Gamma_{L(R) n}(\epsilon)$ is the energy level broadening of the system due to the coupling with the left (right) measuring electrodes, and for simplicity, we consider them constant approximately, i.e., $\Gamma_{L(R) n}(\epsilon)=\Gamma_{L(R)} ; G^{r}$ is the retarded Green's function of the system.

First, for simplicity, we consider an ideal case in which the NS boundary is sharp and clean. Following the previous theory by Demers and Griffin, ${ }^{24}$ which gave a systematic discussion of the anomalous scattering of Bogoliubov excitation at a NS boundary, the energy spectrum of the bound states near the Fermi surface of the SNS structure can be approximately written as

$$
\begin{aligned}
E_{l \pm}(\theta, \phi) & \approx \pm \frac{\hbar^{2} k}{2 m d_{N}}[(2 l+1) \pi+\psi(\theta, \phi)], \\
k & \approx \sqrt{k_{F}^{2}-\mathbf{k}_{\|}^{2}} \approx k_{F} \cos \theta,
\end{aligned}
$$

where, $\quad l=0,1,2,3, \ldots, \quad$ and $\quad \psi(\theta, \phi)=\varphi+\psi_{L}(\theta, \phi)$ $-\psi_{R}(\theta, \phi), \mathbf{k}_{\|}$is the wave vector component parallel to the interface, and the zero point of energy is taken on the Fermi energy $E_{F}$. Here, also for simplicity, we have assumed that the structure of bound states is almost not affected by the fact that the pair potentials may be anisotropic. This assumption is reasonable under the condition $d_{N} \gg \xi_{c}=\hbar^{2} k_{F} / 2 m\left|\Delta_{N}\right|$, which could be satisfied in most cases in the present system. by $^{25}$

Correspondingly, the retarded Green's function is given

$$
G_{l, \pm}^{r}(\epsilon)=\frac{1}{\epsilon-E_{l, \pm}+i\left(\Gamma_{L}+\Gamma_{R}+\Gamma_{S}\right)},
$$

where we have taken into account the energy level broadening $\Gamma_{L(R)}$, and a phenomenological parameter $\Gamma_{S}$ has been introduced to represent the energy level broadening caused by the impurities scattering of the electrons in the metal.

Substituting Eq. (2) into Eq. (1), we get the conductance

$$
\begin{gathered}
g=\int d \mathbf{k}_{\|} g(\theta, \phi), \\
g(\theta, \phi)=-\frac{e^{2}}{h} \int_{-\infty}^{\infty} d \epsilon \frac{\partial f(\epsilon)}{\partial \epsilon} \frac{\Gamma_{L} \Gamma_{R}}{\Gamma_{L}+\Gamma_{R}} \\
\times \sum_{l \pm} \frac{\Gamma_{L}+\Gamma_{R}+\Gamma_{S}}{\left[\epsilon-E_{l \pm}(\theta, \phi)\right]^{2}+\left(\Gamma_{L}+\Gamma_{R}+\Gamma_{S}\right)^{2}},
\end{gathered}
$$

where the sum over the energy states has been done by the integration over $\mathbf{k}_{\|}$and the sum over $l \pm$, and the integration over $\mathbf{k}_{\|}$can be converted to the integration over $\theta$ and $\phi$.

One can define the coherence length of the system corresponding to the energy level broadening as $L_{L(R, S)}$ $=\hbar v_{F} / 2 \Gamma_{L(R, S)}$ and $L=\hbar v_{F} / 2\left(\Gamma_{L}+\Gamma_{R}+\Gamma_{S}\right)$. For the system under consideration, since $d_{N}>L \gg \xi_{c}=\hbar^{2} k_{F} / 2 m|\Delta|$, the sum in Eq. (4b) can be extended to $l=\infty$, yielding 


$$
\begin{aligned}
g(\theta, \phi)= & -\frac{e^{2}}{h} \int_{-\infty}^{\infty} d \epsilon \frac{\partial f(\epsilon)}{\partial \epsilon} \frac{y_{L} y_{R}}{y_{L}+y_{R}} \\
& \times \sum_{\omega_{l}, \pm} \frac{y}{\left(\omega_{l}+\psi \pm \epsilon / E_{0} \cos \theta\right)^{2}+y^{2}},
\end{aligned}
$$

where $\omega_{l}=(2 l+1) \pi, \quad y_{L(R, S)}=d_{N} / L_{L(R, S)} \cos \theta, \quad y=y_{L}$ $+y_{R}+y_{S}$, and $E_{0}=E_{F} / k_{F} d_{N}$. Completing the sum over $\omega_{l}$ in Eq. (5), we obtain the conductance as

$$
\begin{aligned}
g(\theta, \phi)= & -\frac{e^{2}}{h} \int_{-\infty}^{\infty} d \epsilon \frac{\partial f(\epsilon)}{\partial \epsilon} \frac{y_{L} y_{R}}{y_{L}+y_{R}} \\
& \times \frac{\sinh y}{\cosh y+\cos \left(\psi \pm \epsilon / E_{0} \cos \theta\right)} .
\end{aligned}
$$

At zero temperature, it is trivial to finish the integration in Eq. (6) and we obtain

$$
g(\theta, \phi)=\frac{2 e^{2}}{h} \frac{y_{L} y_{R}}{y_{L}+y_{R}} \frac{\sinh y}{\cosh y+\cos \psi(\theta, \phi)} .
$$

For finite temperatures, using a Fourier series expansion,

$$
\frac{\sinh y}{\cosh y+\cos x}=1+2 \sum_{n=1}^{+\infty}(-1)^{n} e^{-n y} \cos n x,
$$

we can complete the integral over $\epsilon$ in Eq. (6) and finally obtain an expression of the $g(\theta, \phi)$ as a Fourier series of $\psi$.

$$
\begin{aligned}
g(\theta, \phi)= & \frac{2 e^{2}}{h} \frac{y_{L} y_{R}}{y_{L}+y_{R}}\left[1+2 \sum_{n=1}^{\infty}(-1)^{n} e^{-n y}\right. \\
& \left.\times \frac{n T / T_{0} \cos \theta}{\sinh \left(n T / T_{0} \cos \theta\right)} \cos n \psi\right],
\end{aligned}
$$

with $k_{B} T_{0}=E_{0} / \pi$.

Using Eqs. (7) and (8), we are able to investigate a number of practical systems. Let us first consider that both superconductors of the SNS structure have isotropic $s$-wave symmetries. In this case,

$$
\psi_{L}(\theta, \phi)=\psi_{R}(\theta, \phi)=0 .
$$

Completing the integral over $\theta$ and $\phi$ in Eq. (4a), one can find that the dominant period component is the $n=1$ term, so that the period of the conductance oscillation with $\varphi$ is $2 \pi$, which is consistent with the experiment. ${ }^{17}$ The calculation result is shown in Fig. 2.

Secondly, we study the anisotropic $s$-wave state. In this case, the order potential can be written as ${ }^{4,11}$

$$
\Delta(\mathbf{k})=\Delta_{0}+\Delta_{1}\left(k_{a}^{2}-k_{b}^{2}\right)^{4},
$$

which is real and positive, and no $\pi$ phase shift exists. So its conductance oscillation will be the same as that in the isotropic $s$-wave case, and does not depend on the misorientation angle $\alpha$.

Thirdly, we investigate the influence of $d_{x^{2}-y^{2}}$ pairing state. When the left superconductor of the structure has the $d_{x^{2}-y^{2}}$ symmetry, the order potential can be written as ${ }^{14}$

$$
\Delta_{L}(\theta)=\Delta_{d}\left(k_{a}^{2}-k_{b}^{2}\right)=\Delta_{d} \cos 2(\theta+\alpha) .
$$

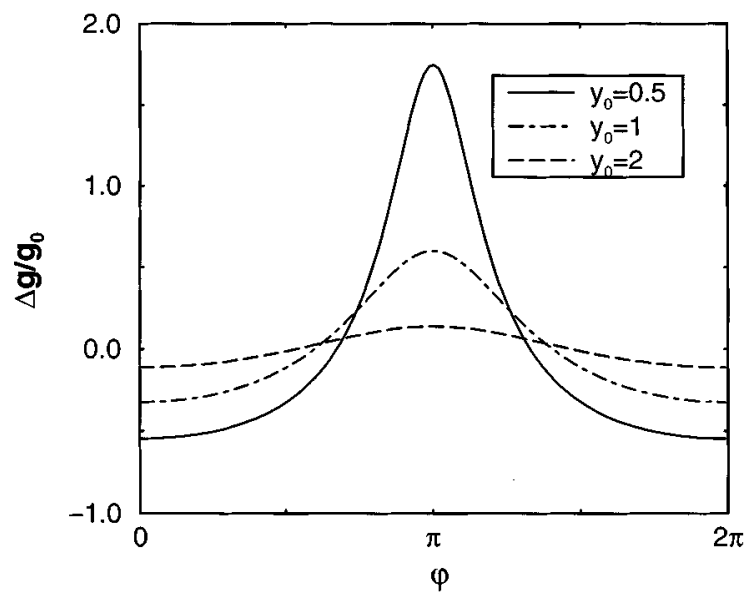

FIG. 2. The phase dependence of the conductance fluctuation normalized to the average conductance for the $s$ wave with different $y_{0}=d_{N} / L$ at $T=0$.

Here, we have considered only that the junction is formed in the $a$ - $b$ plane of the $d$-wave superconductor as shown in Fig. 1. From the previous definition of $\psi_{L}$, we obtain

$$
\psi_{L}(\theta)= \begin{cases}0, & -\frac{\pi}{4}-\alpha<\theta \leqslant \frac{\pi}{4}-\alpha, \\ \pi, & \text { otherwise. }\end{cases}
$$

Completing the integral over $\mathbf{k}_{\|}$in Eq. (4a), it is easy to find that the amplitude of the conductance oscillation depends strongly on the misorientation angle $\alpha$, as shown in Fig. 3. When $\alpha$ varies from 0 to $\pi / 4$, the $2 \pi$ period component decays to zero while the $\pi$ period component remains unchanged. Notice that, Eq. (2) may not be expected when $\theta+\alpha \approx \pi / 4$ since the condition $d_{N} \gg \xi_{c}=\hbar^{2} k_{F} 2 m\left|\Delta_{L}\right|$ is violated; but the error of the integral Eq. (4a) due to this effect is too small to be considered.

More importantly, when $\alpha=\pi / 4$, the electrons injecting to the left SN interface should be divided into two groups, among which an extra $\pi$ phase shift is obtained for those electrons with $0 \leqslant \theta<\pi / 2$. Thus, it can be demonstrated that

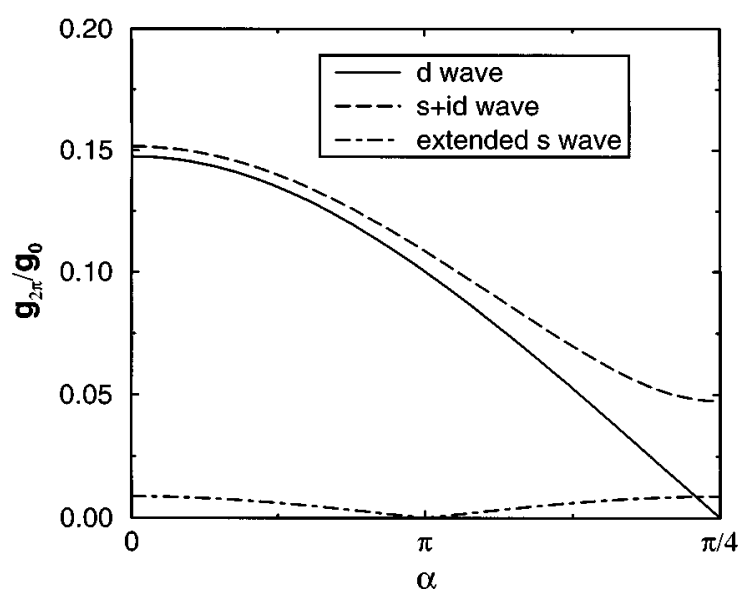

FIG. 3. The $2 \pi$-period component of the normalized conductance fluctuation varies with the misorientation angle $\alpha$ for different paring symmetries, $d$ wave, $s+i d$ wave, and extended $s$ wave at $T=0$ and $y=2$, for the extended $s$ wave $\gamma=0.5$. 
those terms in Eq. (8) with odd $n$ vanish after performing the integral over $\theta$. It means the dominant period component of the conductance oscillation comes from the $n=2$ term, and the oscillation period becomes $\pi$ instead of $2 \pi$ in the conventional case. At the same time, the amplitude of oscillation is strongly suppressed. The similar conclusion can be reached for the case of $s+i d$ pairing as it has been found that $\Delta_{s} \ll \Delta_{d} \cdot{ }^{26}$

As shown in Fig. 2, the manner of conductance oscillation in the $d_{x^{2}-y^{2}}$ state is also different distinctly from that in the $s$-wave case. The most striking difference is that the amplitude of the conductance oscillation depends strongly on the misorientation angle $\alpha$ of the junction in the $d_{x^{2}-y^{2}}$ state, while it is isotropic in the $s$-wave state. Using this feature, it is easier to distinguish the anisotropic $s$-wave state from the $d_{x^{2}-y^{2}}$ state or other phase anisotropic states.

In addition, the extended $s$-wave state is also treated. For this state, its order parameter is usually written as ${ }^{11}$

$$
\Delta(\mathbf{k})=\Delta_{0}\left(1+\gamma^{2}\right)\left[\left(k_{a}^{2}-k_{b}^{2}\right)^{2}-\gamma^{2}\right] .
$$

For $\gamma=0.5$, its conductance oscillation is calculated and the result obtained is shown in Fig. 3, from which we can see that the oscillation amplitude of its $2 \pi$-period component varies also with the angle $\alpha$, but has a different behavior with smaller amplitude. Its amplitude approaches minimum value at $\alpha=\pi / 8$. Therefore, if we make measurements of the conductance at both angles of $\alpha=0$ and $\pi / 4$, it is not difficult to distinguish the extended $s$ wave from the $d$ wave.
In order to obtain a nice and analytical formula for the conductance of the present system, we have assumed the interfaces to be ideal. Notice that nonideal interfaces are always present in the practical systems. We therefore now address the effects of nonideal interfaces. One may define the interfaces scattering Hamiltonian as ${ }^{27}$

$$
H^{\prime}=\sum_{l=L, R} V_{l} \delta\left(z-z_{l}\right)+\sum_{l=L, R} V_{r} f_{l}\left(\mathbf{r}_{\|}\right) \delta\left(z-z_{l}\right)
$$

where $\mathbf{r}_{\|}$is the vector in the $x-y$ plane and $z_{l}$ is the positions of the interfaces $\left(z_{L}=0\right.$ and $\left.z_{R}=d_{N}\right)$. Here the first term describes the scattering by the oxide layer in the interfaces, which has been studied extensively in Ref. 15. Unlike the case of the ideal interfaces, the normal reflecting coefficients do not vanish and the injected electrons will be partly reflected to electrons instead of the holes. Those electrons do not acquire the additional phase and thus have no contribution to the conductance oscillation. The second term of Eq. (9) stands for the scattering due to the rough surfaces and $f\left(\mathbf{r}_{\|}\right)$represents the random surface roughness with $f\left(\mathbf{r}_{\|}\right) \ll d_{N}$. It has been shown in Ref. 27 that this effect could be entirely included in the total reflecting coefficient $R=[(1-k Q) /(1+k Q)]^{2}<1,{ }^{27}$ which implies that a part of the injected electrons are reflected into the incoherent states and have no effect on the interference between the electrons. Taking into account these two effects, we can define the reflecting matrix on each interface as

$$
\mathcal{R}_{L(R)}=\left[\begin{array}{cc}
b_{L(R)} & i a_{L(R)} \exp \left(i \varphi_{L(R)}+i \psi_{L(R)}\right) \\
i a_{L(R)}^{*} \exp \left(-i \varphi_{L(R)}-i \psi_{L(R)}\right) & b_{L(R)}^{*}
\end{array}\right],
$$

where $a_{L(R)}$ is the Andreev reflecting coefficient of the left (right) interface and $b_{L(R)}$ is the corresponding normal reflecting coefficient. The phase factor $\varphi_{L(R)}+\psi_{L(R)}$ for the Andreev reflecting coefficients implies the phase acquired in the abnormal reflecting process. $\left|a_{L(R)}\right|^{2}+\left|b_{L(R)}\right|^{2}=R_{L(R)}$.

Matching the boundary conditions on the two interfaces, one can rederive the quasibound states in the system, and find that

$$
E_{l \pm}(\theta, \phi) \approx \pm \frac{\hbar^{2} k_{F} \cos \theta}{2 m d_{N}}\left[(2 l+1) \pi+\psi_{\mathrm{eff}}\right]-i \Gamma_{I},
$$

$\cos \psi_{\text {eff }}$

$$
\begin{gathered}
=\frac{\left|a_{L} a_{R}\right| \cos \left(\psi+\varphi_{A}\right)-\left|b_{L} b_{R}\right| \cos \left(2 k_{F} \cos \theta d_{N}+\varphi_{N}\right)}{\sqrt{R_{L} R_{R}}}, \\
\varphi_{A}=\arg a_{L} a_{R}^{*} \\
\varphi_{N}=\arg b_{L} b_{R} \\
\Gamma_{I}=\ln \frac{1}{\sqrt{R_{L} R_{R}}} .
\end{gathered}
$$

It is obvious from Eq. (11) that there are two effects coming from the nonideal interfaces: (i) due to the nonvanished normal reflecting probability the phase difference between two superconductors becomes now an effective phase difference $\psi_{\text {eff }}$; (ii) the nonconservation of the reflecting probability caused by the interface roughness leads to an extra broadening of the energy level, which can be absorbed into the general energy level broadening. Therefore, the conductance formulas Eqs. (7) and (8) are still valid in general, but with the renormalized parameters $\psi_{\text {eff }}$ and $y_{\text {eff }}=y_{L}+y_{R}+y_{S}+y_{I}$, where $y_{I}$ represents the level broadening due to the interface roughness.

At zero temperature, substituting Eq. (11) into Eq. (7), we have

$$
\begin{gathered}
g(\theta, \phi)=\frac{2 e^{2}}{h} \frac{y_{L} y_{R}}{y_{L}+y_{R}} \frac{K_{1}}{K_{2}+\cos \left(\psi+\varphi_{A}\right)}, \\
K_{1}=\frac{\sqrt{R_{L} R_{R}}}{\left|a_{L}\right|\left|a_{R}\right|} \sinh y_{\text {eff }}, \\
K_{2}=\frac{\sqrt{R_{L} R_{R}}}{\left|a_{L}\right|\left|a_{R}\right|} \cosh y_{\text {eff }}-\frac{\left|b_{L}\right|\left|b_{R}\right|}{\left|a_{L}\right|\left|a_{R}\right|} \cos \left(2 k_{F} \cos \theta d_{N}+\varphi_{N}\right) .
\end{gathered}
$$




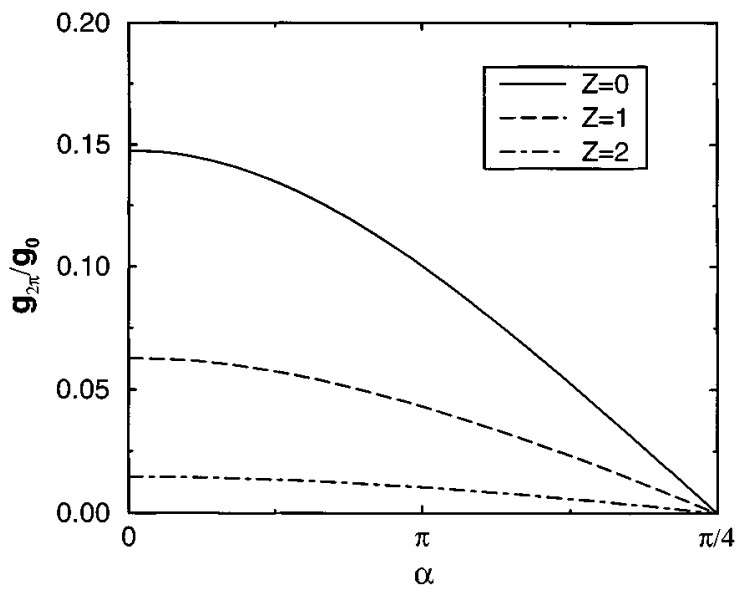

FIG. 4. The $2 \pi$-period component of the normalized conductance fluctuation varies with the misorientation angle $\alpha$ for a $d$ wave superconductor with nonideal interfaces. $Z=2 m V / \hbar^{2} k_{F}$.

This form of the conductance is similar to Eq. (7) except for the renormalized parameters. As a result, the main conclusions reached in the case of the ideal interfaces can still be qualitatively expected in the present case. The results calculated for the interfaces with $\delta$-functional barriers are shown in Fig. 4. It is clearly seen that there is no $2 \pi$-period component either for the $d$-wave case when $\alpha=\pi / 4$ and the only observable effect is the suppression of the oscillation amplitudes. For higher temperature, similar behaviors can be observed from Eq. (8), in which the conductance is now ex- pressed in a Fourier series of the $\cos n \psi_{\text {eff }}$. Obviously, it can be expanded to a Fourier series of the $\cos n \psi$ and thus the conductance can still be expressed in the series of $\cos n \psi$ with the renormalized coefficients. Therefore, we are able to conclude that the existence of the nonideal interfaces has merely a little effect on the conductance and does not change the conclusions reached in the ideal case.

It is well known that a $s$-wave component will be induced near the interface, but the original $d$-wave component is still dominant and can be detected if it indeed exists. In this case, our calculation demonstrates that even for mixed $s+i d$ wave, there is still the conductance oscillation with the misorientation angle $\alpha$, which is clearly different from the pure $s$-wave case. This is because the $\alpha$ dependence of the conductance oscillation is due to an additional intrinsic phase shift existing in the Andreev reflections, which is obviously independent of the amplitude of the superconducting order parameters. ${ }^{24}$

To conclude, we have investigated the conductance oscillations of a mesoscopic normal metal spanning between two superconductors. For the conventional $s$-wave case, results obtained coincide well with the experiment. When one side of superconductors is replaced by a phase-anisotropic one, a phase-dependent conductance oscillation has been found, which could be used to probe the pairing symmetry of high$T_{c}$ superconductors.

This work was supported by the Climing Project of China under Grant No. 85-6 NMS. Z. D. Wang acknowledges the support from the RGC grant of Hong Kong under Grant No. HKU262/95P.
${ }^{1}$ S. E. Barrett, J. A. Martindale, D. J. Durand, C. M. Pennington, C. P. Slichter, T. A. Friedmann, J. P. Rice, and D. M. Ginsberg, Phys. Rev. Lett. 66, 108 (1991).

${ }^{2}$ P. Monthoux, A. Balatsky, and D. Pines, Phys. Rev. B 46, 14803 (1992).

${ }^{3}$ N. E. Bickers, D. J. Scalapino, and S. R. White, Phys. Rev. Lett. 62, 961 (1989).

${ }^{4}$ S. Chakravarty, A. Sudbo, P. W. Anderson, and S. Strong, Science 261, 337 (1993).

${ }^{5}$ G. Kotliar, Phys. Rev. B 37, 3664 (1988).

${ }^{6}$ Q. P. Li, B. E. C. Koltenbah, and R. Joynt, Phys. Rev. B 48, 437 (1993).

${ }^{7}$ D. S. Rokhsar, Phys. Rev. Lett. 70, 493 (1993).

${ }^{8}$ A. G. Sun, D. A. Gajewski, M. B. Maple, and R. C. Dynes, Phys. Rev. Lett. 72, 2267 (1994).

${ }^{9}$ P. Chaudhari and Shawn-Yu Lin, Phys. Rev. Lett. 72, 1084 (1994).

${ }^{10}$ Z. X. Shen, D. S. Dessau, B. O. Wells, D. M. King, W. E. Spicer, A. J. Arko, D. Marshall, L. W. Lombardo, A. Kapitulnik, P. Dickinson, S. Doniach, J. diCarlo, A. G. Loeser, and C. H. Park, Phys. Rev. Lett. 70, 1553 (1993).

${ }^{11}$ D. J. Van Harlingen, Rev. Mod. Phys. 67, 515 (1995).

${ }^{12}$ D. A. Wollman, D. J. Van Harlingen, W. C. Lee, D. M. Ginsberg, and A. J. Leggett, Phys. Rev. Lett. 71, 2134 (1993); A. Mathai et al., ibid. 74, 4523 (1995).
${ }^{13}$ C. C. Tsuei et al., Phys. Rev. Lett. 73, 593 (1994); I. Iguchi and Z. Wen, Phys. Rev. B 49, 12888 (1994); D. A. Brawner and H. R. Ott, ibid. 50, 6530 (1994); C. C. Tsuei et al., Science 271, 329 (1996).

${ }^{14}$ M. Sigrist and T. M. Rice, J. Phys. Soc. Jpn. 61, 4238 (1992); Yukio Tanaka and Satoshi Kashiwaya, Phys. Rev. Lett. 74, 3451 (1995).

${ }^{15}$ Yukio Tanaka, Phys. Rev. Lett. 72, 3871 (1994).

${ }^{16}$ Chia-Ren Hu, Phys. Rev. Lett. 72, 1526 (1994).

${ }^{17}$ P. G. N. de Vegvar, T. A. Fulton, W. H. Mallison, and R. E. Miller, Phys. Rev. Lett. 73, 1416 (1994).

${ }^{18}$ V. T. Petrashov et al., Phys. Rev. Lett. 74, 5268 (1995).

${ }^{19}$ A. F. Andreev, Sov. Phys. JETP 19, 1228 (1964).

${ }^{20}$ B. Z. Spivak and D. E. Khmel'nitskii, JETP Lett. 35, 412 (1982).

${ }^{21}$ R. Landauer, IBM J. Res. Dev. 32, 306 (1988).

${ }^{22}$ M. Büttiker, IBM J. Res. Dev. 32, 317 (1988).

${ }^{23}$ Yigal Meir, Ned S. Wingreen, and Patrick A. Lee, Phys. Rev. Lett. 66, 3048 (1991).

${ }^{24}$ Jacques Demers and Allan Griffin, Can. J. Phys. 49, 285 (1971).

${ }^{25}$ Jun Zang and Joseph L. Birman, Phys. Rev. B 46, 5020 (1992).

${ }^{26}$ Y. Ren, J. H. Xu, and C. S. Ting, Phys. Rev. Lett. 74, 3680 (1995); J. H. Xu, Y. Ren, and C. S. Ting, Phys. Rev. B 52, 7663 (1995).

${ }^{27}$ L. Sheng, D. Y. Xing, and Z. D. Wang, Phys. Rev. B 51, 7325 (1995). 